\title{
On fuzzification of topological categories $]^{*}$
}

\author{
Sergejs Solovjovs \\ Department of Mathematics and Statistics, Faculty of Science, Masaryk University \\ Kotlarska 2, 61137 Brno, Czech Republic \\ solovjovs@math.muni.cz
}

Following the point-set lattice-theoretic (poslat) approach to topology of S. E. Rodabaugh [11, we introduced a more general way to do categorical (fuzzy) topology under the name of categorically-algebraic (catalg) topology [12, which unified many lattice-valued topological settings, and provided the means of intercommunication between different such frameworks. Moreover, motivated by the results of universal topology of H. Herrlich [7, we showed in [13] that a concrete category is fibre-small and topological if and only if it is concretely isomorphic to a subcategory of a category of catalg topological structures, which is definable by topological co-axioms. This achievement relies on the machinery of topological theories of O. Wyler [18, 19], and eventually amounts to the meta-mathematical statement that the whole theory of (fibresmall) topological categories (including, e.g., the categories of poslat topological spaces, shown to be topological over their ground categories in a series of papers of S. E. Rodabaugh) can be done in the framework of catalg topology (a similar but by far more moderate and not that well stated claim was vaguely hinted at in 11 with respect to the poslat topology). The purpose of this talk (which is a shortened version of [14]) is to show a particular development of fuzzy topology in the setting of the catalg one, thereby illustrating the convenient tools of the latter.

There currently exist two popular (in the fuzzy community) approaches to lattice-valued topology (which by no means are the only available). The first one, initiated by C. L. Chang [1] and extended later on by J. A. Goguen [6], defines a many-valued topology on a set $X$ as a subset $\tau$ of an $L$-powerset $L^{X}$ (for some lattice-theoretic structure $L$, e.g., a quantale), which is closed under arbitrary joins and finite meets (or, possibly, quantale multiplication). The second approach, started by U. Höhle [8] and later on generalized independently by T. Kubiak 9 ] and A. Sostak [17, defines a lattice-valued topology on a set $X$ as a map $\mathcal{T}: L^{X} \rightarrow M$ (which employs an additional lattice-theoretic structure $M)$ satisfying the requirements $\bigwedge_{i \in I} \mathcal{T}\left(\alpha_{i}\right) \leqslant$ $\mathcal{T}\left(\bigvee_{i \in I} \alpha_{i}\right)$ for every index set $I$, and $\wedge_{j \in J} \mathcal{T}\left(\alpha_{j}\right) \leqslant \mathcal{T}\left(\wedge_{j \in J} \alpha_{j}\right)$ for every finite index set $J$, which provide lattice-valued analogues of the above-mentioned closure of topology under arbitrary joins and finite meets. In [15, 16, we presented a convenient catalg framework for doing fuzzy topology in the sense of Höhle-Kubiak-Šstak, extending for that purpose the theory of latticevalued algebras of A. Di Nola and G. Gerla [5]. In this talk, we show that the approach to topology of Höhle-Kubiak-Šstak is just an instance of the machinery, which has been called by us the general fuzzification procedure for topological categories. More precisely, given a (fibre-small) topological category $\mathbf{A}$, there exists a topological category $\mathbf{B}$, which contains $\mathbf{A}$ as a full concretely coreflective subcategory, and which can be considered as a fuzzification of A. In particular, the classical category Top of topological spaces (in the role of the above category A) provides an analogue of the original spaces of U. Höhle, the category $L$-Top of Chang-Goguen $L$-topological spaces provides a (partially, variable-basis) analogue of the spaces of Kubiak-Šsostak, whereas the category Loc-Top of [3] gives an analogue of the variable-basis lattice-valued topology in the sense of Höhle-Kubiak-Sostak, which has been recently developed by J. T. Denniston, A. Melton and S. E. Rodabaugh [4].

${ }^{*}$ This research was supported by the ESF Project No. CZ.1.07/2.3.00/20.0051 "Algebraic methods in Quantum Logic" of the Masaryk University in Brno, Czech Republic. 
Strikingly enough, the newly introduced fuzzification procedure for topological categories is additionally well related to the tower extension of topological constructs of D. Zhang 20. (motivated by the buildup pattern of the category of approach spaces of R. Lowen [10, widely used in the lax-algebraic approach to topology of 2]). Moreover, it induces a variable-basis extension of the latter, as well as its "dual" analogue, which (with a suitable starting category in hand) provides the categories, which are isomorphic to the categories of topological spaces in the above-mentioned sense of Höhle-Kubiak-Šostak.

\section{References}

[1] C. L. Chang, Fuzzy topological spaces, J. Math. Anal. Appl. 24 (1968), 182-190.

[2] M. M. Clementino and W. Tholen, Metric, topology and multicategory - a common approach, J. Pure Appl. Algebra 179 (2003), no. 1-2, 13-47.

[3] J. T. Denniston, A. Melton, and S. E. Rodabaugh, Lattice-valued topological systems, Abstracts of the 30th Linz Seminar on Fuzzy Set Theory (U. Bodenhofer, B. De Baets, E. P. Klement, and S. Saminger-Platz, eds.), Johannes Kepler Universität, Linz, 2009, pp. 24-31.

[4] J. T. Denniston, A. Melton, and S. E. Rodabaugh, Interweaving algebra and topology: Latticevalued topological systems, Fuzzy Sets Syst. 192 (2012), 58-103.

[5] A. Di Nola and G. Gerla, Lattice valued algebras, Stochastica 11 (1987), no. 2-3, 137-150.

[6] J. A. Goguen, The fuzzy Tychonoff theorem, J. Math. Anal. Appl. 43 (1973), 734-742.

[7] H. Herrlich, Universal Topology, Categorical topology, Proc. Int. Conf., Toledo/Ohio 1983 (H. L. Bentley, H. Herllich, M. Rajagopalan, and H. Wolff, eds.), Sigma Ser. Pure Math., vol. 5, Heldermann Verlag, 1984, pp. 223-281.

[8] U. Höhle, Upper semicontinuous fuzzy sets and applications, J. Math. Anal. Appl. 78 (1980), 659-673.

[9] T. Kubiak, On Fuzzy Topologies, Ph.D. thesis, Adam Mickiewicz University, Poznań, Poland, 1985.

[10] R. Lowen, Approach spaces - a common supercategory of TOP and MET, Math. Nachr. 141 (1989), 183-226.

[11] S. E. Rodabaugh, Relationship of Algebraic Theories to Powerset Theories and Fuzzy Topological Theories for Lattice-Valued Mathematics, Int. J. Math. Math. Sci. 2007 (2007), 1-71.

[12] S. Solovjovs, Categorically-algebraic topology, Abstracts of the International Conference on Algebras and Lattices (Jardafest), Charles University, Prague, 2010, pp. 20-22.

[13] S. Solovyov, Categorically-algebraic topology versus universal topology, to appear in Fuzzy Sets Syst., http://dx.doi.org/10.1016/j.fss.2012.10.005

[14] S. Solovyov, On fuzzification of topological categories, to appear in Fuzzy Sets Syst., http://dx.doi.org/10.1016/j.fss.2013.05.003

[15] S. Solovyov, Fuzzy algebras as a framework for fuzzy topology, Fuzzy Sets Syst. 173 (2011), no. 1, 81-99.

[16] S. Solovyov, Sobriety and spatiality in categories of lattice-valued algebras, Fuzzy Sets Syst. 204 (2012), 1-26.

[17] A. P. Šstak, On a fuzzy topological structure, Rend. Circ. Mat. Palermo, II. Ser. Suppl. 11 (1985), 89-103.

[18] O. Wyler, On the categories of general topology and topological algebra, Arch. Math. 22 (1971), $7-17$.

[19] O. Wyler, TOP categories and categorical topology, General Topology Appl. 1 (1971), 17-28.

[20] D. Zhang, Tower extension of topological constructs, Commentat. Math. Univ. Carol. 41 (2000), no. $1,41-51$. 